\title{
Supporting Elder Connectedness through Cognitively Sustainable Design Interactions with the Memory Music Box
}

\author{
Rebecca Kleinberger \\ MIT Media Lab \\ Cambridge, MA, USA \\ rebklein@media.mit.edu
}

\author{
Alexandra Rieger \\ MIT Media Lab \\ Cambridge, MA, USA \\ arieger@media.mit.edu
}

\author{
Janelle Sands \\ MIT Media Lab \\ Cambridge, MA, USA \\ jcsands@mit.edu
}

\author{
Janet Baker \\ MIT Media Lab \\ Cambridge, MA, USA \\ janet_baker@email.com
}

\begin{abstract}
Isolation is one of the largest contributors to a lack of wellbeing, increased anxiety and loneliness in older adults. In collaboration with elders in living facilities, we designed the Memory Music Box; a low-threshold platform to increase connectedness. The HCI community has contributed notable research in support of elders through monitoring, tracking and memory augmentation. Despite the Information and Communication Technologies field (ICT) advances in providing new opportunities for connection, challenges in accessibility increase the gap between elders and their loved ones. We approach this challenge by embedding a familiar form factor with innovative applications, performing design evaluations with our key target group to incorporate multi-iteration learnings. These findings culminate in a novel design that facilitates elders in crossing technology and communication barriers. Based on these findings, we discuss how future inclusive technologies for the older adults' can balance ease of use, subtlety and elements of Cognitively Sustainable Design.
\end{abstract}

\section{Author Keywords}

Cognitively Sustainable Design; Technology for Older Adults; Music and Memory; Inter-generational Connection; Familiar Object

\section{CCS Concepts}

-Human-centered computing $\rightarrow$ User centered design; Accessibility theory, concepts and paradigms; Accessibility technologies; Interaction design; Collaborative and social computing; Ubiquitous and mobile computing; Accessibility;

Permission to make digital or hard copies of all or part of this work for personal or classroom use is granted without fee provided that copies are not made or distributed for profit or commercial advantage and that copies bear this notice and the full citation on the first page. Copyrights for components of this work owned by others than ACM must be honored. Abstracting with credit is permitted. To copy otherwise, or republish to post on servers or to redistribute to lists, requires prior specific permission and/or a fee. Request permissions from permissions@acm.org.

UIST'19, October 20-23, 2019, New Orleans, LA, USA.

Copyright (C) 2019 Association of Computing Machinery.

ACM ISBN 978-1-4503-6816-2/19/10 ...\$15.00.

http://dx.doi.org/10.1145/3332165.3347877

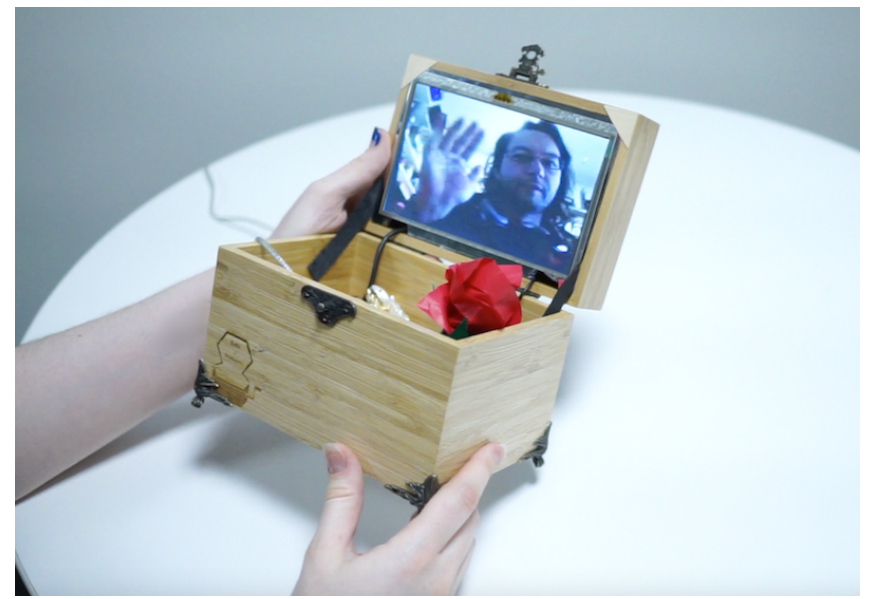

Figure 1. Accessible video call with correspondent from box

\section{INTRODUCTION}

Older adults often experience a lack of connectedness which leads to social isolation $[40,9]$. In recent years, the HCI community has shown interest in designing interventions for individuals facing symptoms related to aging, dementia or general memory loss. We hope to contribute to this conversation through the Memory Music Box (MMB), revealing how technologies that often alienate older generations can be reimagined to help them connect. The Memory Music Box is an interactive system that the elder controls with the natural gesture of opening and closing the box. The Memory Music Box transforms an intuitive everyday object into a portal for video calling and a personalized memory slideshow, to create a sense of connectedness and reminiscence.

Although more connection-support technologies exist than ever before, most social media platforms and ICT systems are not designed with elder-accessibility in mind and present barriers to older populations who are not technologically literate. Even simple platforms pose challenges as components like the touch-screens used in most interfaces require a certain amount of hand moisture that is usually lacking in older skin [18]. Even devices designed for autonomous use by older 
people usually have a figurative "expiry date" as the platforms become increasingly difficult to use as elders age or develop health conditions. Our design is more broadly accessible in light of the fact that one in ten people aged 65 or older is diagnosed with Alzheimer's disease. We accomplish this by employing concepts of Cognitively Sustainable Design - a term we coined which indicates the creation of something so intuitive and accessible that one can continue using it despite an increase in age, disability or cognitive impairment.

Although we as researchers support lifelong learning, we believe that learning should never be a barrier for the simple privilege of connecting with loved ones. The Memory Music Box system is designed to increase social connectedness without technological fluency requirements. If an elder can open a music standard box, they can now connect to their family.

Based upon focus groups, prior research, and interviews, we have identified numerous barriers to connectedness in older adults. The two most prevalent include: technological accessibility and an emotional barrier. Elders feel concern about intruding upon their loved ones' schedules to the extent of ceasing attempts to establish contact. The Memory Music Box addresses both factors through an intuitive design that allows for easy correspondence. Based on early feedback, we selected the form-factor of an old-fashioned music box as it is a neutral, yet familiar object in which older adults tend to keep valuable and sentimental items such as photographs, lockets, medals and trinkets from the past. Brereton and colleagues observed the potential of habituated objects to increase adoption by the older populations [3]. Our work follows aspects of this approach by using the affordances of a familiar object to return control to the user with the goal of increasing their sustained engagement with the device. Within the box is an embedded interactive, connected system, controlled by the simple, natural gesture of opening and closing the box. Secondly, our design uses serendipity to support connectedness. As opening the box delivers a subtle phone notification to the grandchild-user, elders can feel confident that they aren't being bothersome when they reach out. This makes connecting less of a chore, but instead a whimsical and incidental part of life. This idea relates to Wright's work on the role of aesthetics in experience-centered design [44].

A wide range of individuals can benefit from the Memory Music Box. However, the device was initially designed to help individuals of two specific and often intersecting demographics. The first includes elders who have difficulty using existing technologies to connect with their loved ones. We hope that replacing the need to navigate apps on a phone or tablet with the simple gesture of opening a music box, will reduce the technological barrier to feeling connected. Secondly, elders experiencing memory loss and cognitive decline could benefit from the aesthetically pleasing, familiar design, unconsciously associating it with a pleasant experience. This would encourage them to open the box, even if surreptitiously.

Although grandparents and grandchildren were included in our study, it is less of an indication regarding a specific familial relationship. The relationship pairing is mostly in place to provide context in regard to life stage and technological comfort. We also foresee the Memory Music Box device to be potentially beneficial for other forms of pairings or remote pen-pals.

In this paper, we hope to shift the conversation of elder wellbeing to include isolation reduction. We present a holistic approach to targeted design in our collaboration with our user groups. Both older adults and the younger generation aided us in shaping new ICT solutions to improve connectedness. Firstly, we present background information on the social problem of elder isolation, technological barriers in systems designed for the elderly, and a case for the inclusion of music. As presented in the subsequent section, numerous devices exist, but few truly tackle the grandparent-user's need for connection and agency, and of those devices, the ones that do, are laden with technological complexities. As one grandparent (and new iPad owner) exemplified in our focus group "[my family] said I could see the grandkids on here but to be honest, I mostly use it as a paperweight. I can't figure where it turns on or if it needs a plug!" On this basis, we engaged in the research challenge of innovating solutions that can connect grandparent and grandchild-users. We present how we involved these key actors in the design of the Memory Music Box via thoughtful affordances and a simple, engaging user experience design. We also present how we gathered feedback and iterated our design based on an online survey and focus group session. Our findings are presented in an analysis synthesising the results. Finally, we discuss the implications of our findings for further designs, research limitations and innovations for the future.

\section{BACKGROUND}

To situate our work, we consider previous research conducted in the areas of HCI, assistive technology, eHealth, neuroscience, and social psychology.

\section{Loneliness}

Risks of Loneliness

Loneliness and a lack of meaningful connections are now regarded as possible causal factors for faster cognitive decline and the onset or acceleration of dementia and memory loss [43]. A 2006 survey reported that as many as 84 percent of adults over 65 suffer from loneliness in the US [22] Jaremka et al. found higher rates of memory difficulties among breast cancer survivors who experienced high degrees of loneliness [16]. Older individuals with increased feelings of loneliness are also more likely to develop dementia [13]. In contrast, strong and socially meaningful connections were found to be associated with less cognitive decline in older adults $[11,1]$. In light of the findings in the aforementioned studies, it is imperative to focus our efforts on designing technologies that support elder connectedness.

\section{The Social Media Gap}

Current technologies facilitating connection are some of the most ubiquitous and coveted. Social media is among the most prevalent, and young adults participate on numerous networks to stay connected. Although social media has changed the way younger generations connect, older generations are often left out. The gap between younger users and elders grows exponentially as most social networking systems are not designed 
with elders in mind [42]. There are numerous barriers to using these communication technologies for older populations as they have a higher adoption threshold for technology. We see the Memory Music Box portal as a bridge between the two worlds. The grandchild portal allows tech-savvy loved ones to easily share images and music from their social media so elders can stay in the loop.

\section{Long Distance Solutions}

In Social Isolation and Loneliness in Old Age: Review and Model Refinement, [41] Wenger et al. define loneliness as the subjective state of negative feelings associated with perceived social isolation, a lower level of contact than desired or the absence of a specific desired companion. In their Model of Loneliness in Older Adults [8] Fees et al showed feelings of loneliness decrease one's evaluation of physical wellbeing and that the frequency of telephone contact affects loneliness more than the frequency of in-person contact with others. This indicates that increasing the frequency of video calling could have even further beneficial effects on perceived feelings of loneliness.

\section{Technologies Designed for Elders}

\section{One Way vs Two Way Connections}

Existing tech solutions designed in the context of aging, facilitate caregivers in monitoring and tracking patients via offthe-shelf devices such as Keruve [21] an Alzheimer's GPS Tracking Device, or SmartSole [37] a GPS that can be hidden in a shoe and PocketFinder a personal GPS to track your elderly parents on foot or via car. Research ventures such as [26] suggest the use of monitoring devices as well, especially for individuals in rural areas. While loved ones can receive real time data on an elder's whereabouts, we feel that it is important to design for more than a 1-way connection. Although useful for patient safety, these designs fail to answer the needs of older users. We focus on improving the quality of life through the quality of experience. With its precious yet familiar appearance and technological capability, the Memory Music Box is designed to provide meaningful experiences, the sense of reassuring connections.

\section{Technology and Aging}

The other branch of technology designed for elders features digital reminder tools for mild cognitive decline such as the COGNOW day navigator [27] or the Multi-Agent Personal Memory Assistant system [6]. Although momentarily useful, studies reveal [29] that these systems are time bound - unable to provide relevant support as individuals continue to age. Conversely, the MMB is designed to age with the elder via an experience-centered approach. We focus less on assistance and more on creating a sustained sense of wellbeing by integrating accessible connectedness into everyday life. For the last few decades, Electronic Healthcare (eHealth) has focused on designing technology-mediated connections between patients, relatives and healthcare providers $[32,19]$. The challenges tackled by researchers in this domain overlap with ours as they similarly ensure their systems can be navigated by older users. A major difference between our systems lies in the objective. While their system focuses on medication management, our device aims to improve connectedness for the sake of it, not as a way to monitor medication intake.

Other systems that broach elder correspondence have various approaches. The "Family Portrait" project [31] presents an interesting perspective in connecting two households although the system does not support music or video calls. "The Experiences To Go" interface [14] designed in 2013 appears to have laid some of the groundwork in establishing that video conferencing platforms, although vital to long-distance connections, are not sufficient as freestanding interfaces. While their proposed touch-screens pose a difficulty for older hands, the focus on agency concurrently amplifies our design concepts. The MMB incorporates reminiscence components with video aspects while removing the need for touch-activated controls. While myriad platforms have sought to support elder communication, studies reveal that one of the most difficult relationships to maintain (especially long-distance) is the grandparent-grandchild relationship. The paper on "Connecting Grandparents and Grandchildren" [28] further provides detailed insights on how grandparent-grandchild dynamics continue to adapt as life expectancy and communication technologies change. This article identifies that older grandchildren encounter a greater amount of communication difficulty with grandparents, a gap that current technologies do not fully address. Although, the rise in social media has fueled global connectedness for younger generations, it has alienated elders. The 2013 study "Enriching In-Person Encounters Through Social Media: A Study on Family Connectedness for the Elderly" [5] approached this via their Tlatoque system. The interface transfers social media feeds from desktops to a touchscreen digital picture frame device. While elders could see updates through the interface, they could not easily communicate through the platform without prior knowledge or tutorials. Furthermore, using the system required several non-intuitive hand motions to commence operation. Our design builds upon Tlatoque by including "newsfeed"/slideshow elements while removing contact and outreach barriers.

Sometimes, barriers can occur at the point of conversation, "Hello, is Grandma There" [34] focuses on important aspects of intergenerational relationships and how stories or added media formats help to bridge the gap and inspire talking points. This further influenced our platform which allows for music and image sharing via the portal. While aspects of MMB's low barrier functionality are explored above, our work also draws from larger surveys of the design space. The work, "Desiring to be in Touch" [25] provides a broad overview of the landscape of connection challenges faced by older adults. The study sought to "draw a distinction between using technology to mediate intimacy and using it simply for the expression of emotion". The focus groups included "a 30 minute discussion, loosely structured around a number of prompts on topics such as the types of communication media that were normally used, triggers for making contact, and whether there were people who the participants would like more, or less, contact with." The study revealed that Grandchildren (both children and adolescents) were viewed by elders as "too busy for contact." in light of these findings, researchers organised brainstorms alongside their research team which included designers and re- 


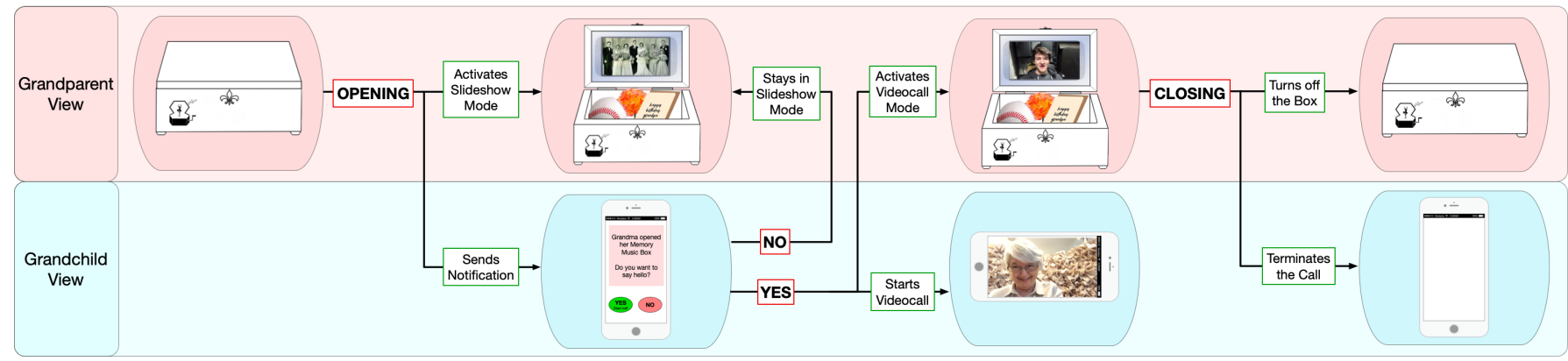

Figure 2. User experience diagram

searchers. The implications which arose from the focus groups provided guidelines for more nuanced and improved connectedness which our design incorporates. The main guidelines identified in the study include: (1) "the importance of communication being personalised", (2) "technologies that allow for a more focused, intense means of communication" and (3) "contact should feel non-intrusive." In regards to personalisation, the MMB focuses on this from the outer shell of the box to the inside. Outside, the box is fully customisable, a canvas awaiting a personal imprint. Inside, the curated slideshow further sparks conversation. We feel the MMB also answers the need for technologies that support "intense, focused communication". Without distracting widgets or complex features, the interaction is as close to an in-person experience, save a lifesize hologram. Thirdly, the MMB is nonintrusive. MMB is careful to respect the privacy of both parties as the screen and camera are only active if it is open and fully in use. While the Family Window (FW) [17] project eliminates formal barriers of initiating long-distance conversation, the continuity of being on camera could be viewed as intrusive. Our work extends this to the older population by creating a system without FWs extensive and possibly complex control mechanisms while also reducing traditional outreach barriers in a nonintrusive manner.

\section{A Case For Inclusion of Music}

\section{Memory}

In designing for elders we feel it would be a mistake to exclude music based upon research revealing the benefits in this population. Music can be used as an anchor point to access personal memories in a beneficial manner and is frequently used in reminiscence therapies [2]. Music engages diverse regions of the brain, particular memories remain preserved and can be recalled upon hearing a familiar melody. According to Janata's fMRI study [15] there is a specific "hub" in the brain that links autobiographical memories to specific songs. This hub is located in the medial prefrontal cortex (MPFC), a region slower to atrophy during the progression of regular aging, Alzheimer's disease or dementia. As people age, and encounter later stage memory loss, music-associated memories in this surviving "hub" are recalled when a particular song is played. As music is like a passcode to the MPFC "memory drive", we felt it was imperative to design our project around it. In doing so, we assessed how music could be used as an anchor point to access personal memories in a beneficial manner.

\section{Music and Connectedness}

The memory music box is not only a music box in form but in the fact that it features a curated playlist accompanying photo gallery elders can hear every time they open the box. According to Ayelet Dassa and Dorit Amir's 2014 study [7], familiar songs increased conversations and connectedness in older individuals. When technologies for elders do incorporate music the experience is often static such as the project Studio Meineck in the UK [20]. Although this project also supports reminiscence, Unlike the Memory Music Box where elders can converse with loved ones, elders do not have an outlet for increased music-triggered conversation.

\section{DESIGN}

The conception and development of the Memory Music Box system was conducted in collaboration with multiple stakeholders. The project was initially sparked following three meetings with behavioural neurologist Dr. Maya Geddes, MD at the Brigham and Women's for Brain/Mind Medicine (CBMM), a clinic specializing in aging. Through observing patients and meeting with clinical staff, the need for accessible interfacing arose. It was noted by clinicians that issues of connectedness continue to be pervasive in aging communities. Throughout our design phases, we consulted with two specialists working at CBMM, Dr. Maya Geddes, MD and neurologist Dr. Michael Erkkinen. In parallel, we worked closely with our elderly collaborators as well as younger researchers who struggle with maintaining long-distance connections with their grandparents. The Memory Music Box emerged from these conversations and presents an answer to challenges outlined in this paper: attenuating isolation by creating connections through improving technological accessibility and reducing emotional barriers.

\section{Interaction Design}

Figure 2 represents an overview of the system through a user experience diagram showing in parallel the grandparent-user and grandchild-user views and interactions. The user actions are shown in red boxes and only consists in opening and closing the box for the grandparent-user, and pressing "yes" or "no" button on a phone for the grandchild-user. Interaction design are detailed in the following subsections.

\section{Grandparent-User}

In focus groups, elders appreciated that the only user input needed is the opening of the box. Upon opening, the box auto- 
matically enters a mode called "slideshow" where a carefully orchestrated series of photos and music will be displayed. The content of the slideshow is curated remotely by the grandchilduser through an online interface.

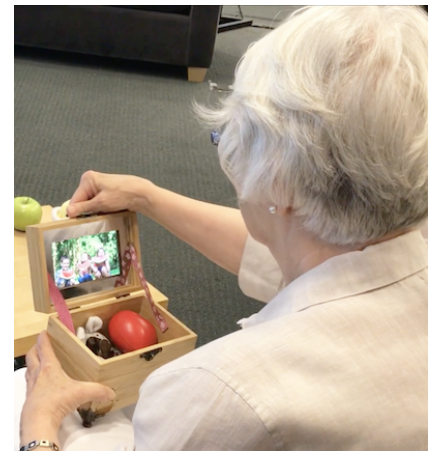

(a) Slideshow interaction

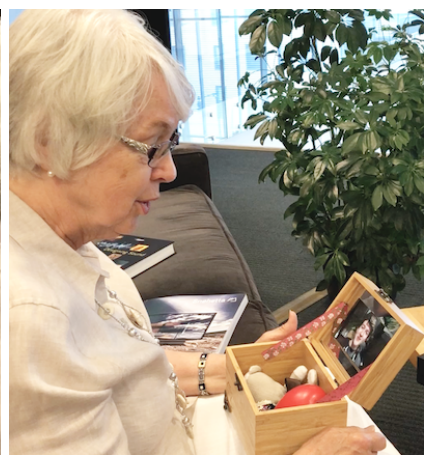

(b) Videocall interaction

Figure 3. Grandparent-user interactions

While entering the slideshow mode (Figure 3.a), the box also sends a discreet phone or email notification to the grandchilduser to inform them that the box is open. If the grandchild is available for a video call, they simply touch the "say hello" icon for the box to switch to call-mode. The grandparent is then automatically connected to the grandchild via videocall (Figure 3.b). The embedded speaker, microphone and camera in the box allow for a seamless video call. The call is stopped when the grandparent-user closes the box or if the grandchild-user ends the call. If the grandchild-correspondent is not connected or not available for a call, the box remains in slideshow mode where the familiar music plays and updated photos of family and friends are displayed on the screen. This interaction is designed such that every opening of the box leads to a feeling of connectedness and comfort, whether the correspondent is available or not.

In addition to its technological interface, the Memory Music Box offers a space to contain important items for the user. From childhood mementos to important written reminders, the box can create a portal of reconnection through real objects allowing the user to explore its contents both in a tangible and digital way.

\section{Grandchild-User}

The interaction requires the grandchildren to be relatively techsavvy as they have to be able to use an email client and be familiar with photo sharing platforms such as Facebook or Instagram. This covers a high proportion of young adults but might exclude young children and young adults with disabilities. Future iterations could explore this further. The grandchild-user has two ways to interact with the device. Asynchronously, they are in charge of curating the content of the slideshow using a password-protected online platform (Figure 4). Their personal account on the platform is uniquely tied to the unique ID of the android system within their grandparent's box for privacy concern. The correspondent can edit music and images at any time, and changes are remotely transferred to reflect in the box. The online correspondent interface also allows them to control the WiFi connection, battery life, and volume of the device.

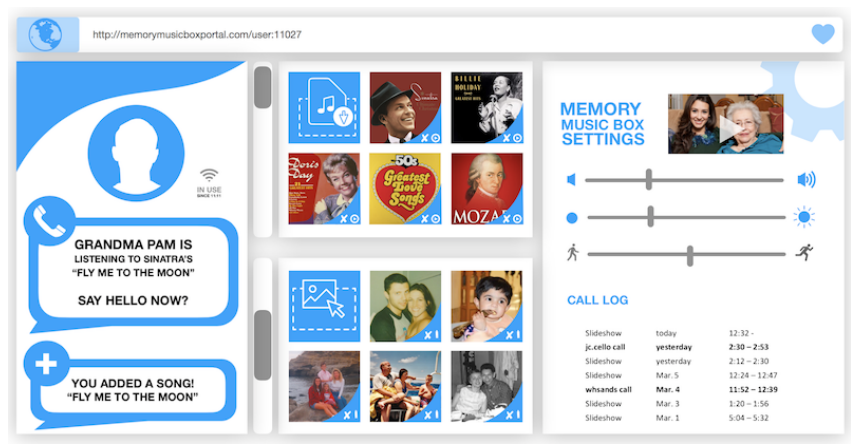

Figure 4. Grandchild-user video call interactions

The second way for the grandchild-user to interact with the box is through video call. We designed the box interaction to ensure that calls can only be initiated by the grandparentuser to provide them with agency and control over the box interaction. Each time the box is opened, the grandchild-user receives a subtle email or text notification on their phone informing them that the box is open (Figure 5.a). They can decide to ignore the notification if they are unable to answer or they can press a button that automatically connects them to the grandparent through the calling feature (Figure 5.b).

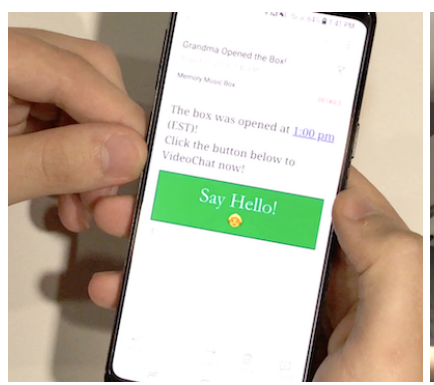

(a) Notification reception

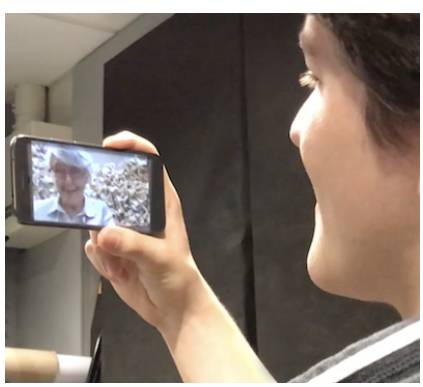

(b) Videocall interaction
Figure 5. Grandchild-user video call interactions

\section{System Design}

\section{First Prototype}

We developed an early stage prototype (diagram Figure 6) using an embedded Linux computer (raspberry pi), in a box specifically built to fit the electronics. The Raspberry Pi was connected to a USB microphone, a 5' touchscreen, a Pi camera and a mini speaker connected through an 1/8th inches cable. The speaker was attached to the bottom part of the box to use the resonance of the container and increase the overall volume. All the hardware was hidden behind a one-sided mirror frame. A pressure sensor was used to detect if the box was opened. This original system used Python to sense the interaction and launch the two modes. The video call mode was implemented using the google hangout API and the slideshow mode was made using Pyglet [12] and Pygame [36] libraries.

The first prototype featured selected design elements and aesthetic detailing ensuring that the box could be recognized as 


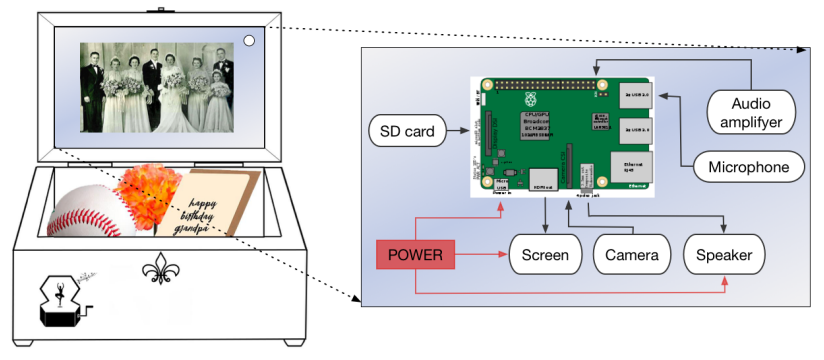

Figure 6. First prototype system diagram

a piece of craftsmanship. The designs give the impression that the box was hand-made especially for the user. This perpetuates an atmosphere of appreciation for the object. We used bamboo for the box and antique brass for the decorated feet, hinges, and latch. The top of the box is laser etched with a design personalized to the taste of the recipient. This design was used as a tangible object to discuss and review user interactions with a team of designers and physicians. The prototype offered good flexibility in terms of development and choice of hardware. The modularity allowed us to go through a series of implementations to quickly iterate the interface and experience design based upon feedback.

\section{Second Prototype}

Once we selected specific interaction components, we decided to implement the system on an Android-based platform. Indeed, using a cheap Android device and turning its processor into a Raspberry-like board gave us the same flexibility, comparable performance, memory, storage and all the I/O necessary for a fraction of the cost. The activation-trigger pressure sensing function was replicated by using the front camera as a light sensor to detect when the box is in use. Our current prototype is entirely embedded within an Android device, placed horizontally behind a mirrored frame in the lid of a hand-crafted, old-style music box (diagram Figure 6). The device is mounted to ensure that the front-facing camera and microphone are in an optimal position. As all 'buttons' are inaccessible behind the frame, unintended interactions cannot occur. The front-facing camera, speakers, microphone, and screen are employed during slideshow and call modes, while position sensors allow the box to respond to being opened or closed. More compact, elegant and tamper-proof, this design direction renders our system more easily deployable for the future as an affordable kit. We envision that this "off-the-shelf" kit could simply contain an empty neutral box (to be decorated in DIY-fashion), the mounting frame and a personal pin and URL to download the application to any available phone.

The software was built using Java and relies on the Skype for Business API for call services. It seamlessly transitions between an active mode when the box is opened and a sleeping mode when the box is closed, while the system periodically reads light sensors from the front-facing camera to determine whether the box has reopened, it portrays a dark screen with no audio. Upon opening the box, the application immediately joins a unique video call in the background, but the camera and microphone remain off while the user enjoys a fullscreen slideshow of photos, accompanied by music. The application

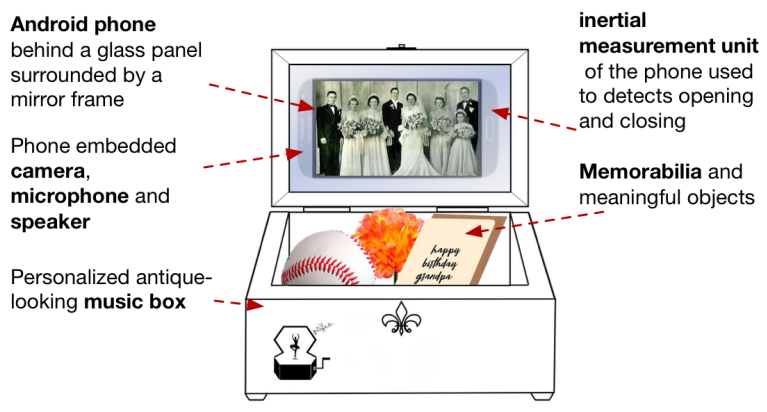

Figure 7. Second prototype system diagram

automatically transitions between slideshow and call modes depending on whether the other person is present in the unique video meeting. The video call is self-contained within the application and is protected by all the privacy and security protection supported within the Skype platform.

This design and set-up prioritize simplicity to cater to grandparent-users at different levels of physical and cognitive abilities. Considering grandchild-users' tech-savviness and attempting to maximize connectivity, this prototype features a grandchild-user notification system in which grandchild-users are alerted when the box opens with an email to their personal account. They can follow an active video call link within that email to join the call in the Skype for Business application on their own phone or computer. With its scalability for multiple boxes, its simplicity and reliability for grandparent-users when encountering weak internet connections, this prototype offers exciting improvements in deployability and connectivity.

\section{EVALUATION}

Our two target groups were grandparent-users $(\mathrm{GpU})$ and grandchild-users (GcU). While $\mathrm{GpU}$ focus groups were conducted in person, GcU surveys were completed through a secure online survey. Both focus groups facilitated our participatory approach to the design refinement of the Memory Music Box. Throughout the conception and development processes, we iterated our design choices to maximize the adoption potential of the device for older adults. The next section presents a formal assessment of our design in comparison to existing frameworks through the lens of elder technology adoption.

\section{Technology Adoption Assessment for Older Adults}

In order to evaluate the Memory Music Box system, we implemented Lee's adoption factors presented in [24]. The ten identified adoption factors that predict older adults and adoption of technology are: experience, usability, emotion, social support, value, confidence, affordability, accessibility, technical support and independence. These factors are also aligned with the work of Suh et al. featuring six constructs that predict user burden with computer systems [38]. The presented categories are revealed to stymie initial adoption of technological systems ( 1 - difficulty of use, 2 - physical, 3 time and social, 4 - mental and emotional, 5 - privacy, and 6 financial). For each of the ten adoption factors, we analysed and challenged our design choices to understand the adoption potential for older adults. 


\section{EMOTION}

This factor relates to our concept of Cognitively Sustainable Design. By creating a device that encourages emotional attachment, the interactive system can remain relevant for the user even as cognitive and physical abilities decline. Based upon prior research [30] users have a higher probability of projecting emotional qualities onto our system as it is personalised. Still, some might argue that a stuffed toy or robotic form factor could also reach this goal.

\section{VALUE}

The Box is designed as a personalisable artifact to be gifted by the GcU. Thus, it is likely to carry a higher affective value in addition to its functionality. The outside appearance can be embellished through collaborative crafting sessions with grandparents and correspondents, further increasing its value.

\section{EXPERIENCE}

The system inside the box might feel foreign to the $\mathrm{GpU}$ but the outside appearance invites interaction and functions analogously with other familiar systems like a regular jewelry or music box. No further knowledge is required for the user to benefit from the MMB. Once opened, it runs entirely by itself. When trying to interact with a laptop, it is not the opening of the laptop that stops elderly adults but what happens after.

\section{AFFORDABILITY}

The design choices of the prototype will allow researchers to reduce production costs as the MMB only requires three key elements: a freely downloadable application, a low-cost android phone and an empty box with the right inset dimensions. If the MMB device were to be deployed on a larger scale, researchers envision releasing codes, guidelines and plans on an open source platform with a tutorial for the grandchild.

\section{USABILITY}

The interaction is simple and excludes superfluous features. This helps ensure older users or elders with physical and cognitive limitations are not challenged to attain technologically fluency before use. In spite of this, if the MMB's context is forgotten (due to cognitive impairment) or not explained during acquisition, elders could be confused with its functionality. In the future we will explore approaching this by adding a short reminder message before the slideshow plays.

\section{CONFIDENCE}

Contrary to most current devices, there is no need to fear unknowingly pushing a wrong button as there are no buttons on our system. One could even introduce the box to a user by saying that there are no right or wrong ways to use it. Additionally, the device is designed to empower the $\mathrm{GpU}$ with the agency to initiate calls.

\section{SOCIAL SUPPORT}

Contrary to the very individual experience of talking on the phone, the MMB can be shared with others physically present around the user. Focus group participants expressed interest in sharing their MMB's with friends to learn more about one another. While the current screen might be too small for several people to effectively interact with the $\mathrm{MMB}$, other design variations could be explored.

\section{ACCESSIBILITY}

The device is designed to be gifted to the $\mathrm{GpU}$ to reduce acquisition barriers. There is no need for the user to have extensive prior knowledge of the device itself. We hope to make the designs publicly available along with a simple tutorial targeted to $\mathrm{GcU}$ population. Using open-source code, an old phone and an existing box they can easily create the experience. While accessibility could become an issue in case of severe physical limitation, researchers are exploring further adaptations.

\section{TECHNICAL SUPPORT INDEPENDENCE}

In the current use scenario, technical support would be assured by the grandchild user. From the online platform, GcUs have access to information such as wifi signals, volume, luminosity, battery level, and the time and length of each interaction. This will allow GcUs to not only identify possible technical problems but also be proactive when noticing that the box is not being used. The system presents a straightforward interface for any relatively tech-savvy grandchild users who are comfortable with computers.

Apart from keeping the battery of the box sufficiently charged grandparent users can tend to the use and care of the box independently as long as they have moderate dexterity. In designing the box, we aimed to increase the feeling of agency and engagement. Indeed, contrary to existing digital frames that continuously display images, our device requires an active control action from the user. This design choice was guided by the decision to keep agency central to the experience.

To summarise, the affordances of the Memory Music Box bypass most of the factors that are shown to decrease adoption barriers. Researchers led focus groups and conducted a survey to gather feedback from our two target populations $(\mathrm{GpU}$ and $\mathrm{GcU}$ ) based on the aforementioned ten adoption factors.

\section{Grandparent-User - Focus group}

\section{Focus Group Methodology}

To learn about target user-group applications of the Memory Music Box, we collaborated with the Hebrew Senior Life Center in Brookline, MA, an independent senior living facility. The format of a focus group was chosen as a platform to co-design the MMB. Researchers sought potential user feedback and first impressions to shape usage, flow and aesthetics. Elders at the center were told in advance about the workshopformat focus group and registered to attend. The co-ed group included 10 individuals recruited through flyers and advertisements by the center. Our participant group included 8 women and 2 men, all between the ages of 70 and 95 . The participants were divided in three groups of 3-4 participants and group discussions were guided by two researchers respectively. In each group, participants had the opportunity to interact with the Memory Music Box during the session and were invited to discuss three themes of interaction: connection, memories and usability. The length of the session was a total of an hour and a half during which participants were presented with a selection of drinks and refreshments. Researchers opened with a musical prelude and a welcome introduction before merging into the main part of the interaction. The focus group yielded positive feedback from participants following user-interaction experiences as well as general insights and suggestions to increase accessibility.

While the current study has been conducted with healthy older adult populations per our initial Institutional Review Board (IRB) clause, future iterations will be sparked by a broader array of individuals with diverse abilities with the goal of codesigning increasingly accessible MMB's. In the future, we plan on involving differently-abled populations in testing the device including children with disabilities and older adults with early stage Alzheimer's disease. 
The workshop featured four main parts: introduction, questionnaire, interaction, and assessment. As participants arrived they were greeted and presented with consent forms, one helper from the center and four researchers were present to answer any questions. Upon the start of the session, elders were shown a video of the Memory Music Box's functions and features. The narrated video revealed a grandparent and grandchild using the box to communicate in a narrative everyday setting. Following the introduction of the concept, researchers shared their enthusiasm to learn from participant interactions and experiences with the Memory Music Box. Following this, participants were asked to separate into smaller groups. The focus group segment of the workshop was arranged with a head researcher and an assisting undergraduate researcher present at each table. Researchers led the focus groups with interactive Memory Music Box prototypes and a questionnaire developed based upon accessible design research [33], assessments charts of devices for elderly individuals $[35,24]$ and previous work on assessing connectedness $[39,10]$.

\section{Focus Group Questionnaire}

The questionnaire featured two distinct portions: preexperience and post-experience. Before interacting with the Memory Music Box, we sought to learn about the participants' current state of connectedness. To assess this, the first portion of the questionnaire posed detailed questions about current correspondences with grandchildren/loved ones and overall satisfaction rates. Although one listener indicated that they did not have friends/relatives outside the center, all focus group participants stated that they had specific friends or relatives with whom they enjoyed connecting. Although all participants had some form of contact with their correspondents, 80 percent of the participants expressed dissatisfaction with the frequency of connection. None of the participants possessed the technological experience necessary to video-conference with their loved ones via Skype or any alternative platforms and therefore were unable to communicate with their relatives in real-time with the exception of participant number 6 . This participant's family installed Facebook live notifications on her phone so she could see her loved ones at the push of a button but could not interact with them, nor could they see her. To learn more about the rate of dissatisfaction and lack of connection, we posed the question "What makes it difficult to connect with your friends/family?" A range presented across responses, however, outstanding concerns included the busyness of their correspondents (as participants hesitated to reach out in fear of interrupting full schedules) and technological challenges. 100 percent of participants stated that current technologies did not support their connectedness with their correspondents with one participant specifying that although some technologies could be helpful, there is a lot of room for improvement. The post-experience sessions included questions about the subject's overall impression of the device, projected frequency of use and expected reception by their grandchild-correspondent. During the sessions, researchers at their respective tables asked each participant the intended questions and took note of the answers on prepared charts. Charts included rating scale systems so participants responses could be synthesised into quantifiable information alongside qualitative quotations. Following the sessions, results were analysed to ascertain general views of potential GpUs.

\section{Focus Group Interaction}

Following the first part of the questionnaire, participants interacted with prototypes of the Memory Music Box. Participants were able to pass the box around, video-conference with another researcher and experience a slideshow of music and images. Each participant took the time to explore the box's features and demo the experience firsthand. Following the interactive session, researchers asked participants about their thoughts. Some of the responses include: "I think this is wonderful", "This would be great to have", "It reminds me of Skype but much more accessible" as well as accessibility questions such as: "Wonderful, I'd love to have one to share with others. Could the screen size be bigger/brighter and the volume higher?" and "I love the easy to handle system. Maybe it could be lighter to open for arthritic hands."

\section{Focus Group Design Feedback}

The focus group not only helped to confirm many components of our design but also supported specific changes in the latest prototype and online interface. For instance, participants mentioned that they would prefer the system to be preset on the highest possible microphone and speaker volume. The positioning angle of the lid was satisfactory for most participants but not all. Further data analysis will help to inform weather creating a click-based adjustable angle positioning system could improve usability. One participant mentioned that adding a large size handle on the lid could help low dexterity elders to better enjoy the interaction. Another focus group participant said that she "would love this on the nightstand". In a future version we would like to include an automatic blue tone reduction on the display if the system detects that the box is being opened at night to prevent circadian rhythm disruption. Congruently, the box could automatically select more relaxing music from the playlist.

\section{Focus Group Findings}

A notable finding arose from the focus group: elders saw immense potential for two distinct kinds of connectedness leveraged through the Memory Music Box: 1) Interfamilial Connectedness and 2) Interpersonal Connectedness. Interfamilial Connectedness was currently unsatisfactory for $80 \%$ of the participants due mostly to respect for loved ones busy schedules and a lack of accessible technologies. Participants expressed that they felt their connections would improve with the box as it is an accessible device that sends subtle and nonintrusive alerts to their point-of-contact allowing outreach to be unobtrusive - therefore, leading to frequent connections. The second form, Interpersonal Connectedness relates to sharing one's personally curated Memory Music Box slideshow with peers and friends inside the senior living center. Reminiscence Therapy [4] research reveals that both music and pictures can support autobiographical memories, making it easier to recall and share life-events in conversation. Many of the participants noted that they would enjoy sharing their memory boxes to learn more about one another, deepening conversations to include life-experiences and current family updates. 


\section{Feedback for Future Work}

Although our novel design has many beneficial aspects, our team looks forward to further developing the MMB. The design thus far has been hewn through a myriad of stakeholder input, constructive feedback and evaluations at various intervals in the prototyping process. In awareness of the efforts that have allowed us to develop the MMB to this extent, we also acknowledge the potential for growth and improvement. Overall, we received overwhelmingly positive feedback from the focus group. In parallel there were a few negative but highly relevant comments from participants highlighted within this section. When the focus group event began, a few potential participants disclosed that they did not wish to join the study. When asked why, the individuals commented that they were either "not interested in technology" or lacked technological fluency. None of these individuals saw the MMB but seemed to shy away from the mere terminology before the chance of interaction. There were no tests conducted where an elder simply opened the MMB without knowing what would be inside. Instead, all participants were primed and aware of the functionality prior to interaction. Perhaps an emphasis on the music box element could spark a more open-minded and exploratory user-feedback session. This inspires us to consider reformatting future study experiences so that the word 'technology' will not be an additional barrier. Further comments are as follow:

"This would be great for people with accessibility challenges. If someone has only coarse motor skills left, could they even open the box?" This concern was raised by a focus group participant who was a retired accessible designer. She noted that although she felt the form-factor would be accessible for numerous conditions, there are some which would require the box to have a more easily graspable handle. Fortunately, the box is fully customisable and grandchild-users or local caregivers could help to affix a handle, tether or similar assistive modality as needed. In spite of this, we are considering future user testing to identify a system of adaptive modular grips that can be snapped into place.

"What if the 'grandchild' person I want to talk to is also technologically illiterate?" Although our current design aims to address the generation gap, future iterations could support boxto-box contact features. Initial plans for this system include similar low-threshold "plug-and-play" usage alongside nonintrusive notifications via a gentle "glow" when a connected MMB is opened.

"Could the screen size be bigger/brighter and the volume higher?" While the grandchild is able to adjust brightness and volume settings, screen size is limited by the box itself. In some cases, a larger screen size could be beneficial although it would increase the size of the MMB, which is currently no bigger than a traditional music box. However, having learned of this point of interest, we anticipate exploring sizing options in future prototype testing.

"I frequently see friends and family in person, they all live nearby!" One of the participants remarked the above comment stating that both his friends and relatives lived nearby and visited often. As creators of the MMB, we acknowledge that there will be cases where the MMB may not be required due to its target audience. For this participant, perhaps the box is only a gateway to exploring technology as it is not needed for connectedness. Researchers were glad to hear of this participant's frequent interactions with loved ones while simultaneously acknowledging that many elders are separated from their relatives. It is our hope that the Memory Music Box can act as a bridge; bringing connections and good memories in places of isolation.

\section{Grandchild-User - Online Survey}

To gather feedback from potential GcU, we conducted an online survey regarding current interactions with the grandparent, general feedback on the device, and suggestions regarding the online interface. For this portion of the study, we selected the format of an online survey over an in-person study, due to our consideration of time constraints. We feel this decision is justified as our device specifically targets young, busy individuals for the "grandchild" category. The online component ensured that they could be included. Furthermore, as the MMB's GcU interface is computer based, an online survey is closer to the actual interaction. Thirty-one young adults aged between 18 and 54 years of age recruited through student mailing lists participated in the survey.
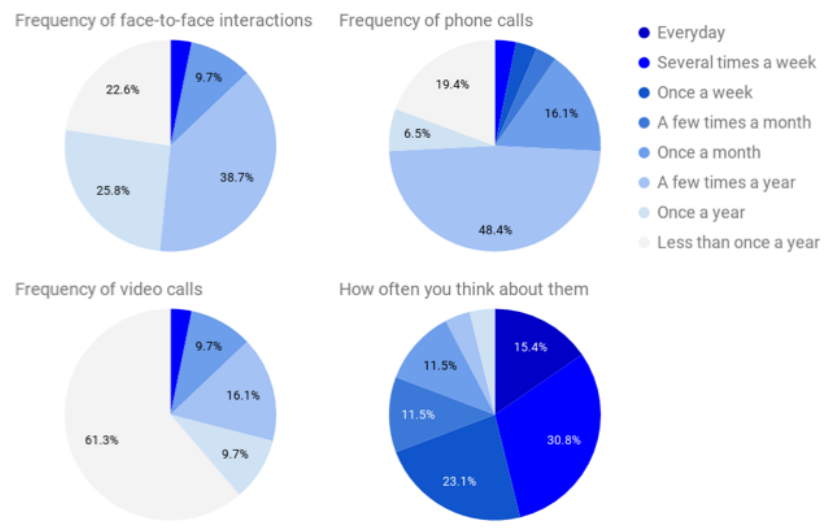

Figure 8. Frequencies of current interactions

\section{Current Interactions with Grandparents}

The survey revealed limited interactions with grandparents compared to how often the participants were thinking about them (see Figure 8). $87 \%$ of participants stated that they only visited their grandparents a few times a year or less. $74 \%$ of participants stated that they only called their grandparent on the phone a few times a year or less; this number was $87 \%$ for video calls. When asked who initiates the calls, $45 \%$ responded that phone calls are always initiated by themselves, $31 \%$ answered that it is $50 / 50$. $60 \%$ of GcUs responded that when video calls occur, they are always self-initiated.

Across all answers, communication was reported to last from five minutes to an hour, and the content of the conversations generally stays at a superficial level $(52 \%)$ despite being very caring (52\%) (see Figure 9). Most people revealed not knowing what music their grandparent enjoys (74.19\%) and even more have the impression that their grandparent does not know 


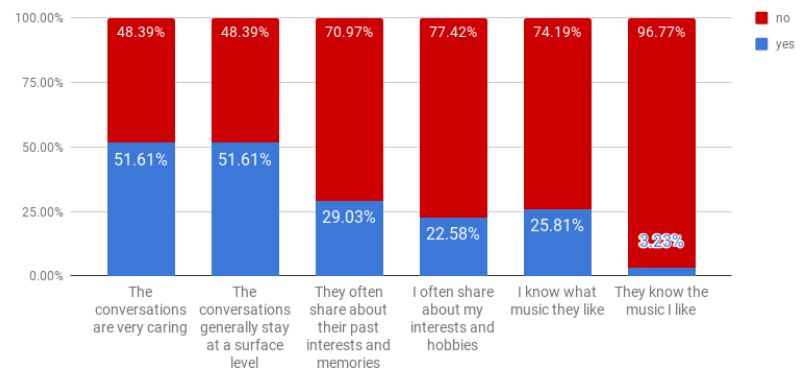

Figure 9. Content of current interactions with grandparent

about their musical taste $(96.77 \%)$. Only about a third of participants reported that their grandparent often shares about their past interests and memories $(29.03 \%)$ and only a quarter of grandchildren reported frequent sharing of their own interests and hobbies (22.58\%).

When asked if any factors limit their grandparent's ability to connect, $40 \%$ answered that memory loss is an obstacle for connection, $36 \%$ mentioned physical difficulties to use a device, $24 \%$ reported that their grandparent did not own a phone or computer. $80 \%$ stated that their grandparent experienced general difficulties with using technologies (see Figure 10).
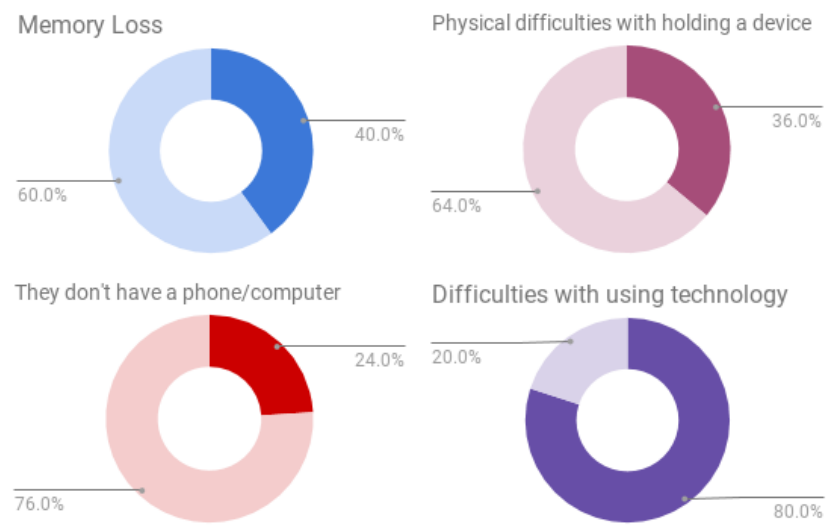

Figure 10. Factors limiting the connection, lighter color means "yes" and darker color means "no"

Globally, only $32.3 \%$ of Grandchild participants reported feeling happy with current interactions with their grandparents and only $38.7 \%$ of Grandchild participants felt that their Grandparent is satisfied with the current interactions they share.

\section{General feedback on the device}

After asking about current information, the participants were introduced to the Memory Music Box device through an introductory text and a short video. Following this, they were asked to provide feedback (see Figure 11).

Most found the device interesting and desirable for their own interactions with their grandparents and we gathered feedback such as "it seems great!! and really easy for them to use", "Very cool!", "looks really awesome!", "I love it", "easy to use form-factor for older generations, auto-connected which is convenient", "it is very cute and it could make a lot of people very happy" and "It would definitely be helpful for facilitating communication between me and my grandparents", "My

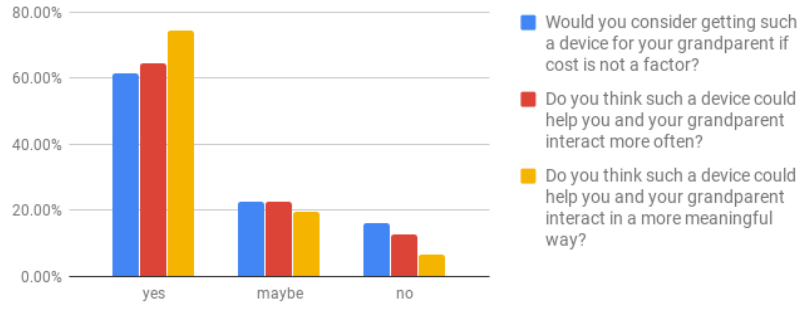

Figure 11. General thoughts on the Memory Music Box device

grandpa would love something like this!" Many believed that the device would improve frequency of contact (See Figure 14), however a small percentage questioned whether the box format would be beneficial. One respondent stated that they "think it is interesting. It requires good internet connection. If it doesn't work a few times my grandmother might get frustrated." The design of the box proved not to be ideal for everyone with a small number of participants saying "Seems ok. Does not feel that different from a tablet." The conceptual idea of incorporating reminiscence therapy into technology to spark GpU memories was quite popular as $74 \%$ agreed doing so could produce more meaningful conversations.

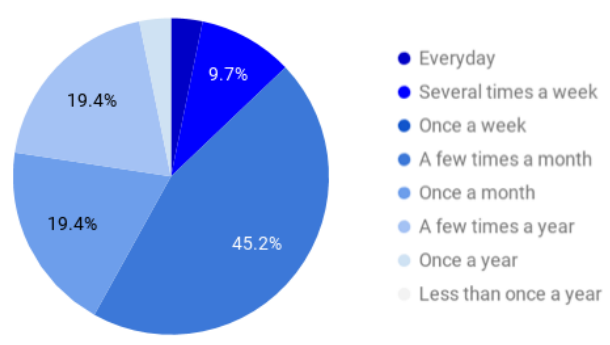

Figure 12. Projected frequency of content updates

\section{Feedback on the Grandchild Interface}

When asking participants how frequently they would update the media content on the box, $3.23 \%$ answered they would do so everyday, $9.68 \%$ answered several times a week, $45.16 \%$ answered "several times a month", $19.35 \%$ said "about once a month" and $23 \%$ answered "a few times a year" (see Figure 12).

We also asked participants how user-friendly they think the box would be for their grandparent. Most participants felt the ergonomics of the device would be user-friendly for their grandparent as well as the online interface for themselves (see Figure 13).

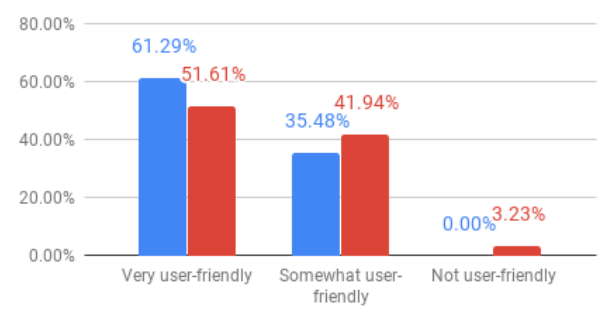

How user-friendly do you think the box would be for your grandparent?

Do you think such a device could help you and your grandparent

Figure 13. On user-friendliness 
Finally we asked grandchild participants how often they think their grandparent might open the box if they had such a device. About half of all participants thought that their grandparent would open the box everyday and $87 \%$ felt that their grandparent would open the box at least once a week.

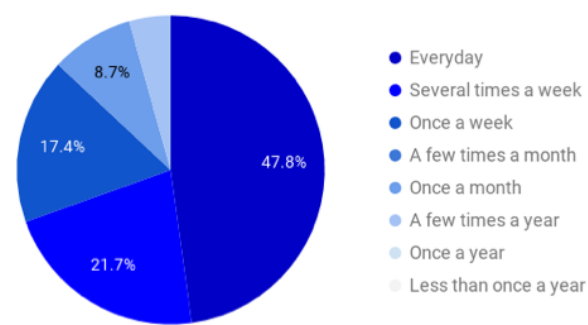

Figure 14. Projected frequency of grandparent interactions

\section{Insights}

Several grandchild participants worried that such a device could create a heavy commitment and they would feel guilty not answering to notifications or forgetting to update photos and music often enough. Indeed, we received comments such as "I would feel guilty not picking up if I were busy, knowing they were looking at pictures of me", or "It's a very sweet interaction, but I'm afraid if the grandchild doesn't answer, the grandparent will be sad. But maybe it's ok because if they see new photos or hear new music, they would know that I thought about them." However, in regard to feedback obtained from the focus group with older adults, grandparents are generally highly aware of their grandchild's busy schedules as it tends to prevent grandparents from initiating phone or video calls. Moreover, grandparents from the focus group expressed happily spending a lot of time looking at a limited set of photographs. Even a minor improvement on this end such as an extra photograph or two a year would be welcomed.

Another major insight from the survey is how dissatisfied grandchildren are with their current interactions with grandparents. Only a third indicated satisfaction with their current interactions. In addition, we observed an important disparity between how often grandchildren think about their grandparent and how rarely they actually call/ video call or have face-toface interactions with them. We compared these frequencies with the reported projected frequencies at which grandchildren would update the photos and music on the box. More than three quarters of participants reported that they would update media content at least once a month (77\%) which is three times more than people who reported calling their grandparent at least once a month $(25 \%)$, and about six times more than phone calls and six times more than participants who reported video-calling (13\%) or having face-to-face interactions (13\%) with their grandparent at least once a month. This insight reinforces the idea that cognitively sustainable devices, such as the Memory Music Box, might help bridge the gap between how little grandchildren interact with their grandparents and how often they think about one another.

When looking at the content of current interactions, more than half of the grandchild participants indicated that discussions often remained at a superficial level, and some seemingly important details about each other's lives, such as musical tastes, are not often discussed. As few as $4 \%$ of grandparents seem to know what music their grandchild listens to and only about a quarter of grandchildren know their grandparent's musical taste. More generally, only $30 \%$ of grandparents share about their past, interests and memories while $23 \%$ of grandchildren share about their interests and hobbies. Both the focus group and the online study revealed a real desire for ways to trigger more meaningful and personal interactions. One focus group participant explained "for music there is a generational divide. I mainly listen to classical music and country but they listen to very different things that I don't relate to. They call it 'Phish' I think, I would be interested in listening to what they like, maybe we could understand more of each others, if they could share it with the box it could be fantastic". Similarly, grandchildren seemed hopeful that well curated triggers could improve not only the quantity but also the quality of interactions with their grandparent as $74 \%$ answered that they believe such a device could help them and their grandparent interact in a more meaningful way. Here, music acts as a language that can unexpectedly express unknown identities and further explain ones that are known, which in hand, deepens social connections. In future work, these factors need to be tested longitudinally. A further step would include performing an evaluation with individuals suffering from memory loss and dementia within the context of sustained MMB use.

Some study participants expressed concerns regarding their grandparents using the box: "Love it, but I hope they can actually use it without problems". Others were worried that lack of infrastructure on their grandparents end would affect the usability: "it is a nice concept and would make it easier for my grandparents to call me. However, I would need to setup wifi for them at their home (they don't have wifi)", "Very interesting concept. Would like to know how robust it is in different circumstances (i.e., have you considered an alternative to a no-WIFI environment where Skype calls are not feasible)." Based upon these results, we feel the creation of a non-wifi iteration is an important avenue for future work. While most participants felt the Memory Music Box would be an ideal device for their grandparents, a few participants noted that their grandparents were already technologically fluent and wouldn't require the ease of access provided by the MMB: "It's cool. My grandparents are very competent with technology (they are on Facebook, know how to use smartphone, etc). They have some pride surrounding the issue. It's possible that they might consider it slightly condescending." Both concerns noted in the feedback (no wifi and technology proficiency) reveal two extreme ends of their respective spectrums, shining light on the specificity of the population that will benefit from the box.

A major objective in our qualitative study was to examine how grandparents experience the empowering potential of being an actor in personalised ICT solutions. While elders have responded with overwhelmingly positive feedback, we remain aware that our design could seem inadvertently limiting or patronising if an extremely tech-fluent grandparent desires to have further control of the system. In spite of this, the 
Memory Music Box can still be a pleasant artifact - far more interactive than a digital picture frame (which even tech-savvy individuals enjoy) and furthermore could help to increase confidence with more complex technologies. However, at its core, our device is meant for users who feel helpless with existing Information and Communication Technologies (ICT) and are seeking easier alternatives while keeping a level of independence in the process.

\section{CONTRIBUTIONS}

When approaching the challenge of creating a design for the aging population, we acknowledge the intersectional individualities that accompany this age group. Unlike younger target populations, elders frequently experience rapid changes in their physical and cognitive capabilities as well as their living situations. For example, an elder aging in-place with their family may be moved to a skilled nursing facility where healthcare takes precedence over connectedness. Although creating a one-size-fits-all design proves unlikely in these situations, our development of the terminology "Cognitively Sustainable Design" presents an answer. In spite of dramatic life changes, we envision the Memory Music Box will remain a familiar object in an elders domestic space. While further longitudinal evaluations with the MMB will enable us to hone design elements, we wish to bring the novel concept of "Cognitively Sustainable Design" to the HCI conversation.

We envision HCI as a space through which to address gaps in opportunity for older generations. Moving beyond monitoring, the Music Memory Box focuses exclusively on empowering agency and facilitating meaningful connections. The design is uniquely poised to appeal to a diverse user base due to low threshold usability features as well as a design which encourages continued use through later years due to MMB's Cognitive Sustainability. Our novel approach ensures that accessibility is forged through intuitive design, implementing technology within a familiar platform already common within the domestic space. The Memory Music Box presents a system for serendipitous communication between older and younger generations while also mediating curated images and music, encouraging reminiscence and connectedness.

More broadly, our work fits in the rhetoric around target population empowerment in HCI [23]. We believe that our findings are a call to a broader discussion about the use of HCI to empower elders who are not familiar with technology, especially through the lens of facilitating connectedness. On one hand, any solution that does not tackle this problem frontally could add more layers of complexity between the two individuals trying to connect which could obscure the root problem by simply addressing symptoms. Indeed, many technologies designed "for" older adults are often aimed at providing reassurance to caregivers. These interventions often omit the perspective of the older adult and fail to probe the long-term impact of the technology on their mutual relationship. On the other hand, as the shift in technology has been so important these last few decades, many born after the sixties have acquired a sufficient technological literacy that could allow them to keep up more efficiently with future advances. Our elders today could be the last generation of humans to feel completely disconnected from technology (at least in most developed countries and urban environments), as researchers we believe we have the duty to support them nonetheless.

\section{CONCLUSION}

There are many approaches to supporting elders with new innovations. While there are numerous areas needing technological intervention, research in the fields of aging and empathic design reveals that loneliness and isolation are major contributors to a rapid decline in cognitive function. In spite of this, many innovations do not support the connectedness with which so many elders struggle. In the context of Cognitively Sustainable Design, the Memory Music Box is proposed as a novel contribution to the discussion around familial isolation, and builds on previous work in this space within the interface research community. The MMB speaks to the broader design-research and positive-ageing communities about the importance of empowerment, autonomy, and self-expression through the personalisation and application of design objects by older individual users, especially those experiencing cognitive impairment. Our findings confirm that the current state of connectedness for older individuals, especially those in care facilities, is waning during this time of technological revolution. Based upon our surveys, focus groups and user tests analyzed throughout this paper, we propose that the Memory Music Box has the potential to fill an existing gap in the wellbeing of elderly individuals by facilitating communication.

Ideally, there should not be a major learning curve in order to achieve the simple task of talking to a family member. Although there are many systems with a higher learning threshold, we envision our device as a low-barrier gateway for individuals to become more comfortable with technology. Positive interactions can instill elders with agency and in some cases the confidence to expand their circle of technologies to include more complex interfaces.

Whether or not one expands their technological horizons, the box provides immediate access. We can foresee a future where the correspondent can select different interaction levels in the box so elders can expand their technological capabilities within their comfort-zones. We envision the Memory Music Box as something with which older individuals can age. Crafted within the principles of Cognitively Sustainable Design, even as memory loss increases and mobility decreases, the box will remain a familiar and intuitive object with which elders can continue interacting.

Although the MMB fills an important gap, we can envision other populations who could similarly benefit from this device. Specific minor changes in the form factor and usage flow could allow individuals with developmental disabilities, reduced cognitive or motor function and young children to engage with the MMB as well.

Although the purpose of our work is currently geared to elders, there is great opportunity in the exchange of information, legacy and wisdom for the younger individual: a kind of guidance and mentorship that youth in all of its haste is seldom privy to. From our data with the "young correspondents/grandchild" group, grandchildren are seeking a deeper 
connection with their elders. We refer to those advanced in years as sages and elders because of their wisdom hewn from decades of living; garnered knowledge from life experiences and major events impacting our world.

Non-western cultures often look to the oldest members of their communities for guidance. We pose the question, what has happened to our elders? Why are they so often far away in care facilities, segregated from the general population without the chance for information transfer? Our elders need us as we need our elders. Sadly due to the connection fallout, it is almost as though we have established two different planets: one planet that uses technology and one that doesn't. Often we cast the one without technology in a pejorative light, considering the people there to be "old fashioned" or obsolete. However this is not a factor of elders falling behind, this is an issue of equity, the weight of which we feel upon our shoulders. The Memory Music Box would afford a low-cost, equal-access platform to facilitate meaningful connections across these divides.

\section{ACKNOWLEDGEMENT}

The authors would like to thank the residents of the Hebrew Senior Life Center in Brookline, MA for their excitement and collaboration, and all the participants who shared their time and stories as well as the authors' respective grandparents for their inspiration. We would also like to thank Akito van Troyer, Sara Sime and George Stefanakis for their help in preparing the focus group interactions. Finally we wish to thank our PI Tod Machover and our lab group for encouragement in our pursuit of this field of study. We also acknowledge the research ethics board of our institution for reviewing and approving the use of human participants for this research protocol.

\section{REFERENCES}

[1] Shari S Bassuk, Thomas A Glass, and Lisa F Berkman. 1999. Social disengagement and incident cognitive decline in community-dwelling elderly persons. Annals of internal medicine 131, 3 (1999), 165-173.

[2] Susan Bluck and Linda J Levine. 1998. Reminiscence as autobiographical memory: A catalyst for reminiscence theory development. Ageing \& Society 18, 2 (1998), 185-208.

[3] Margot Brereton, Alessandro Soro, Kate Vaisutis, and Paul Roe. 2015. The Messaging Kettle : Prototyping Connection over a Distance between Adult Children and Older Parents. Proc. CHI 2015 (2015), 713-716. DOI : http://dx.doi.org/10.1145/2702123.2702462

[4] Kai-Jo Chiang, Hsin Chu, Hsiu-Ju Chang, Min-Huey Chung, Chung-Hua Chen, Hung-Yi Chiou, and Kuei-Ru Chou. 2010. The effects of reminiscence therapy on psychological well-being, depression, and loneliness among the institutionalized aged. International Journal of Geriatric Psychiatry: A journal of the psychiatry of late life and allied sciences 25, 4 (2010), 380-388.

[5] Raymundo Cornejo, MóNica Tentori, and JesúS Favela. 2013. Enriching in-person encounters through social media: A study on family connectedness for the elderly.
International Journal of Human-Computer Studies 71, 9 (2013), 889-899.

[6] Ângelo Costa, Paulo Novais, Ricardo Costa, Juan Corchado, and José Neves. 2010. Multi-agent personal memory assistant. Trends in practical applications of agents and multiagent systems (2010), 97-104.

[7] Ayelet Dassa and others. 2014. The role of singing familiar songs in encouraging conversation among people with middle to late stage AlzheimerâĂŹs disease. Journal of music therapy 51, 2 (2014), 131-153.

[8] Bronwyn S Fees, Peter Martin, and Leonard W Poon. 1999. A model of loneliness in older adults. The Journals of Gerontology Series B: Psychological Sciences and Social Sciences 54, 4 (1999), P231-P239.

[9] Graeme Hawthorne. 2006a. Measuring social isolation in older adults: Development and initial validation of the friendship scale. Social Indicators Research 77, 3 (2006), 521-548. DOI : http://dx.doi.org/10.1007/s11205-005-7746-y

[10] Graeme Hawthorne. 2006b. Measuring social isolation in older adults: development and initial validation of the friendship scale. Social Indicators Research 77, 3 (2006), 521-548.

[11] Catherine Helmer, D Damon, L Letenneur, C Fabrigoule, P Barberger-Gateau, S Lafont, R Fuhrer, T Antonucci, D Commenges, JM Orgogozo, and others. 1999. Marital status and risk of AlzheimerâĂŹs disease A French population-based cohort study. Neurology 53, 9 (1999), 1953-1953.

[12] A Holkner. 2008. Pyglet: Cross-platform windowing and multimedia library for Python. Google Code (2008).

[13] Tjalling Jan Holwerda, Dorly JH Deeg, Aartjan TF Beekman, Theo G van Tilburg, Max L Stek, Cees Jonker, and Robert A Schoevers. 2012. Feelings of loneliness, but not social isolation, predict dementia onset: results from the Amsterdam Study of the Elderly (AMSTEL). $J$ Neurol Neurosurg Psychiatry (2012), jnnp-2012.

[14] Kori Inkpen, Brett Taylor, Sasa Junuzovic, John Tang, and Gina Venolia. 2013. Experiences2Go: sharing kids' activities outside the home with remote family members. In Proceedings of the 2013 conference on Computer supported cooperative work. ACM, 1329-1340.

[15] Petr Janata. 2009. The neural architecture of music-evoked autobiographical memories. Cerebral Cortex 19, 11 (2009), 2579-2594.

[16] Lisa M Jaremka, Juan Peng, Robert Bornstein, Catherine M Alfano, Rebecca R Andridge, Stephen P Povoski, Adele M Lipari, Doreen M Agnese, William B Farrar, Lisa D Yee, and others. 2014. Cognitive problems among breast cancer survivors: loneliness enhances risk. Psycho-Oncology 23, 12 (2014), 1356-1364. 
[17] Tejinder K Judge, Carman Neustaedter, and Andrew F Kurtz. 2010. The family window: the design and evaluation of a domestic media space. In Proceedings of the SIGCHI Conference on Human Factors in Computing Systems. ACM, 2361-2370.

[18] Tobias Kalisch, Jan-Christoph Kattenstroth, Rebecca Kowalewski, Martin Tegenthoff, and Hubert R Dinse. 2012. Cognitive and tactile factors affecting human haptic performance in later life. PLoS One 7, 1 (2012), e30420.

[19] Anne Marie Kanstrup, Sergi Rotger-Griful, Ariane Laplante-Lévesque, and Annette Cleveland Nielsen. 2017. Designing connections for hearing rehabilitation: Exploring future client journeys with elderly hearing aid users, relatives and healthcare providers. In Proceedings of the 2017 Conference on Designing Interactive Systems. ACM, 1153-1163.

[20] Fares Kayali, Naemi Luckner, Oliver Hödl, Geraldine Fitzpatrick, Peter Purgathofer, Tanja Stamm, Daniela Schlager-Jaschky, and Erika Mosor. 2013. Elements of play for cognitive, physical and social health in older adults. In Human Factors in Computing and Informatics. Springer, 296-313.

[21] Keruve. 2008. Keruve Alzheimer's GPS Tracking Device. (2008). http://www. keruve.com/

[22] William Lauder, Kerry Mummery, Martyn Jones, and Cristina Caperchione. 2006. A comparison of health behaviours in lonely and non-lonely populations. Psychology, Health \& Medicine 11, 2 (2006), 233-245.

[23] Amanda Lazar, Raymundo Cornejo, Caroline Edasis, and Anne Marie Piper. 2016. Designing for the third hand: Empowering older adults with cognitive impairment through creating and sharing. In Proceedings of the 2016 ACM Conference on Designing Interactive Systems. ACM, 1047-1058.

[24] Chaiwoo Lee and Joseph F Coughlin. 2015. PERSPECTIVE: Older adults' adoption of technology: an integrated approach to identifying determinants and barriers. Journal of Product Innovation Management 32, 5 (2015), 747-759.

[25] Siân E Lindley, Richard Harper, and Abigail Sellen. 2009. Desiring to be in touch in a changing communications landscape: attitudes of older adults. In Proceedings of the SIGCHI Conference on Human Factors in Computing Systems. ACM, 1693-1702.

[26] Hung-Huan Liu, Yung-Ju Chen, Yu-Jen Chang, and Wei-Hsun Chen. 2009. Mobile guiding and tracking services in public transit system for people with mental illness. In TENCON 2009-2009 IEEE Region 10 Conference. IEEE, 1-4.

[27] FJ Meiland, Annika Reinersmann, Birgitta Bergvall-Kåreborn, David Craig, Ferial Moelaert, Maurice Mulvenna, Chris Nugent, Tony Scully, Johan Bengtsson, and R M Dröes. 2007. COGKNOW: development of an ICT device to support people with mild dementia. Journal on Information Technology in Healthcare 5, 5 (2007), 166-177.

[28] Karyn Moffatt, Jessica David, and Ronald M Baecker. 2013. Connecting grandparents and grandchildren. In Connecting Families. Springer, 173-193.

[29] Kellie Morrissey, John McCarthy, and Nadia Pantidi. 2017. The Value of Experience-Centred Design Approaches in Dementia Research Contexts. In Proceedings of the 2017 CHI Conference on Human Factors in Computing Systems. ACM, 1326-1338.

[30] Ruth Mugge, Jan PL Schoormans, and Hendrik NJ Schifferstein. 2009. Emotional bonding with personalised products. Journal of Engineering Design 20, 5 (2009), 467-476.

[31] Elizabeth D Mynatt, Jim Rowan, Sarah Craighill, and Annie Jacobs. 2001. Digital family portraits: supporting peace of mind for extended family members. In Proceedings of the SIGCHI conference on Human factors in computing systems. ACM, 333-340.

[32] World Health Organization. 2015. World report on ageing and health. World Health Organization.

[33] Anne Marie Piper, Ross Campbell, and James D Hollan. 2010. Exploring the accessibility and appeal of surface computing for older adult health care support. In Proceedings of the sigchi conference on human factors in computing systems. ACM, 907-916.

[34] Hayes Raffle, Glenda Revelle, Koichi Mori, Rafael Ballagas, Kyle Buza, Hiroshi Horii, Joseph Kaye, Kristin Cook, Natalie Freed, Janet Go, and others. 2011. Hello, is grandma there? let's read! StoryVisit: family video chat and connected e-books. In Proceedings of the SIGCHI conference on human factors in computing systems. ACM, 1195-1204.

[35] Marcela D Rodríguez, Victor M Gonzalez, Jesus Favela, and Pedro C Santana. 2009. Home-based communication system for older adults and their remote family. Computers in Human Behavior 25, 3 (2009), 609-618.

[36] Pete Shinners and others. 2011. PyGame-Python game development. Retrieved from 785 (2011).

[37] SmartSole. SmartSole:hidden, wearable monitoring and recovery solution for wandering. (????).

http://gpssmartsole.com/gpssmartsole/

[38] Hyewon Suh, Nina Shahriaree, Eric B Hekler, and Julie A Kientz. 2016. Developing and validating the user burden scale: A tool for assessing user burden in computing systems. In Proceedings of the $2016 \mathrm{CHI}$ conference on human factors in computing systems. ACM, 3988-3999.

[39] Daniel T van Bel, KCHJ Smolders, Wijnand A IJsselsteijn, Yvonne de Kort, and others. 2009. Social connectedness: concept and measurement. Intelligent Environments 2 (2009), 67-74. 
[40] David J. Weeks. 1994. A review of loneliness concepts, with particular reference to old age. International Journal of Geriatric Psychiatry 9, 5 (1994), 345-355. DOI : http://dx.doi.org/10.1002/gps. 930090502

[41] G Clare Wenger, Richard Davies, Said Shahtahmasebi, and Anne Scott. 1996. Social isolation and loneliness in old age: review and model refinement. Ageing \& Society 16, 3 (1996), 333-358.

[42] Joseph Wherton, Paul Sugarhood, Rob Procter, and Trisha Greenhalgh. 2015. Designing technologies for social connection with older people. Aging and the Digital Life Course 3 (2015), 107.

[43] Robert S Wilson, Kristin R Krueger, Steven E Arnold, Julie A Schneider, Jeremiah F Kelly, Lisa L Barnes, Yuxiao Tang, and David A Bennett. 2007. Loneliness and risk of Alzheimer disease. Archives of general psychiatry 64, 2 (2007), 234-240.

[44] Peter Wright, Jayne Wallace, and John McCarthy. 2008. Aesthetics and experience-centered design. ACM Transactions on Computer-Human Interaction (TOCHI) 15, 4 (2008), 18. 